POS PROCEEDINGS

\title{
Semileptonic B decays
}

\section{Giulia Ricciardi}

Dipartimento di Fisica, Universitá degli Studi di Napoli Federico II, Via Cintia, 80126 Napoli, Italy and INFN, Sezione di Napoli, Via Cintia, 80126 Napoli, Italy

E-mail: giulia.ricciardiena.infn.it

We review recent progress in exclusive $B \rightarrow D^{(*)}$ and inclusive $B \rightarrow X_{u}$ semileptonic $\mathrm{B}$ decays.

Xth Quark Confinement and the Hadron Spectrum,

October 8-12, 2012

TUM Campus Garching, Munich, Germany 


\section{Introduction}

In the last years, detailed studies of semi-leptonic decays of $B$ mesons have been made possible by the vast samples of $B$ mesons recorded at CLEO and, especially, at the B factories. Also D $\varnothing$ and LHCb have helped, collecting data on $B$ mesons decays into excited $D$ meson states and $B_{s}$ decays. Ultimate precision should be reached by future SuperB and Belle II facilities.

Semi-leptonic decays play a critical role in the analysis of the unitarity triangle. Information about the CKM matrix elements $\left|V_{c b}\right|$ and $\left|V_{u b}\right|$ is provided by exclusive and inclusive processes driven by $b \rightarrow c(u) l v_{l}[1,2]$. We can compare, as an example, with leptonic decays $B^{+} \rightarrow l^{+} v$. The decay rates depend on the product of the modulus of the $\left|V_{u b}\right|$ and of the decay constants $f_{B}$, therefore the determination of the CKM element requires the theoretical knowledge of $f_{B}$. In addition, the comparison between experimental and theoretical results presents some hardships. Experimental disadvantages come from the helicity suppression of the leptonic decays and the comparatively high rate of the process $B^{+} \rightarrow l^{+} v \gamma$. For decays into tauons, these effects appear to be small, while the helicity suppression is mostly broken. Moreover, the existence of a charged Higgs boson (or any other charged object beyond the Standard Model) would easily modify the decay rates. Exclusive semileptonic decays can be called to the rescue by supplying complementing informations. They are not helicity suppressed, still they retain the possibility of theoretical simplification by factorizing the leptonic current. They are tree level dominated and new physics is generally not expected (at least to a high degree of accuracy).

Here we review recent progress in exclusive $B \rightarrow D^{(*)}$ and inclusive $B \rightarrow X_{u}$ semileptonic B decays.

\section{Exclusive $B \rightarrow D^{(*)}$ decays}

The differential ratios for the semileptonic decays $\bar{B} \rightarrow D l \bar{v}$ and $\bar{B} \rightarrow D^{*} l \bar{v}$ depend on several unknown form factors, that are constrained and reduced in the heavy quark limit. Neglecting the charged lepton and neutrino masses, we can write

$$
\begin{aligned}
\frac{d \Gamma}{d \omega}(\bar{B} \rightarrow D l \bar{v}) & =\frac{G_{F}^{2}}{48 \pi^{3}}\left(m_{B}+m_{D}\right)^{2} m_{D}^{3}\left(\omega^{2}-1\right)^{3 / 2}\left|V_{c b}\right|^{2}(\mathscr{G}(\omega))^{2} \\
\frac{d \Gamma}{d \omega}\left(\bar{B} \rightarrow D^{*} l \bar{v}\right) & =\frac{G_{F}^{2}}{48 \pi^{3}}\left(m_{B}-m_{D^{*}}\right)^{2} m_{D^{*}}^{3}\left(\omega^{2}-1\right)^{1 / 2} \chi(\omega)\left|V_{c b}\right|^{2}(\mathscr{F}(\omega))^{2}
\end{aligned}
$$

Here $\mathscr{F}(\omega)$ and $\mathscr{G}(\omega)$ are the form factors in the heavy quark limit, $\chi(\omega)$ is a known phase space factor and $\omega=v_{B} \cdot v_{D^{(*)}}=\left(m_{B}^{2}+m_{D^{(*)}}^{2}-q^{2}\right) / 2 m_{B} m_{D^{(*)}}$ is the product of the heavy quark velocities $v_{B}=p_{B} / m_{B}$ and $v_{D^{(*)}}=p_{D^{(*)}} / m_{D^{(*)}}$. Let us observe that outside the heavy quark limit $\mathscr{F}(\omega)$ contains a combination of three form factors (one vector and two axial vectors) related to the three $D^{*}$ helicity states. In the $B$ rest frame, $\omega$ corresponds to the energy of $D^{(*)}$ normalized by its mass, that is to $\omega=E_{D^{(*)}} / m_{D^{(*)}}$. The values of $\omega$ are constrained by kinematics: $\omega \geq 1$, with largest value $\omega \simeq 1.5$. At the nonrecoil point, $\omega=1$, heavy quark symmetries play a useful role in setting the normalization $\mathscr{F}(1)=\mathscr{G}(1)$; this point, however, is not directly accessible experimentally due to the kinematic suppression in Eqs. (2.1). The main theoretical problem is the non-perturbative evaluation of the form factors. By exploiting Eqs. (2.1), the product $\left|V_{c b}\right| \times$ form factor can be extracted by experimental fits to the differential decays rates. 
At present the $\bar{B} \rightarrow D^{*} l \bar{v}$ decay is measured with a better accuracy than the $\bar{B} \rightarrow D l \bar{v}$ decay, therefore the exclusive determination of $\left|V_{c b}\right|$ relies on the theoretical non-perturbative results for the form factor $\mathscr{F}$. The most recent the Heavy Flavor Averaging Group (HFAG) experimental fit [3] gives

$$
\left|V_{c b}\right||\mathscr{F}(1)|=(35.90 \pm 0.45) \times 10^{-3}
$$

The only unquenched result available $[4,5]$ gives

$$
\mathscr{F}(1)=0.908 \pm 0.017
$$

including the enhancement factor 1.007, due to the electroweak corrections to the four-fermion operator mediating the semileptonic decay. By taking Eqs. (2.2) and (2.3), the latest value of $\left|V_{c b}\right|$ reads [3]

$$
\left|V_{c b}\right|=\left(39.54 \pm 0.50_{\exp } \pm 0.74_{\mathrm{th}}\right) \times 10^{-3}
$$

where the errors come from experiment and QCD lattice calculations, respectively. The $\mathscr{F}(1)$ form factor has also been calculated recently using zero recoil sum rules. The quoted value [6, 7], including full $\alpha_{s}$ and up to $1 / m_{5}^{2}$, is

$$
\mathscr{F}(1)=0.86 \pm 0.02
$$

By using the same Eq. (2.2) in combination with Eq. (2.5), one obtains

$$
\left|V_{c b}\right|=\left(41.6 \pm 0.6_{\exp } \pm 1.9_{\mathrm{th}}\right) \times 10^{-3}
$$

an higher value, in average and in theoretical error, than the one given by the lattice calculation.

Let us compare the previous determinations with the $\left|V_{c b}\right|$ value extracted from $\bar{B} \rightarrow D l \bar{v}$ decays. The most recent unquenched calculation dates back to 2005 [8] and gives, after correcting by the usual factor of 1.007

$$
\mathscr{G}(1)=1.074 \pm 0.024
$$

which, using the latest HFAG average [3], that includes older Aleph, CLEO and Belle measurements, as well as the new 2008-2009 BaBar data, becomes

$$
\left|V_{c b}\right||\mathscr{G}(1)|=(42.64 \pm 1.53) \times 10^{-3}
$$

The resulting estimate is

$$
\left|V_{c b}\right|=\left(39.70 \pm 1.42_{\exp } \pm 0.89_{\text {th }}\right) \times 10^{-3}
$$

in good agreement with the lattice determination from $\bar{B} \rightarrow D^{*} l \bar{v}$ Eq. (2.4). However, the experimental error is more than twice larger. An alternative lattice determination, currently available only in the quenched approximation, consists in calculating the form factor normalization directly at values $\omega>1$, avoiding the large extrapolation to $\omega=1$ and thus reducing the model dependence $[9,10]$. This approach, by using $2009 \mathrm{BaBar}$ data [11], gives a slightly higher value than the lattice result (2.9), that is

$$
\left|V_{c b}\right|=\left(41.6 \pm 1.8 \pm 1.4 \pm 0.7_{F F}\right) \times 10^{-3}
$$


The errors are statistical, systematic and due to the theoretical uncertainty in the form factor $\mathscr{G}$, respectively. The most recent non lattice calculation combines the heavy quark expansion with a "BPS" expansion [12] giving in this limit

$$
\mathscr{G}(1)=1.04 \pm 0.02
$$

With such estimate one finds [13]

$$
\left|V_{c b}\right|=\left(40.7 \pm 1.5_{\exp } \pm 0.8_{\text {th }}\right) \times 10^{-3}
$$

in agreement, within the errors, with both lattice determinations (2.9) and (2.10).

Until a few years ago, only decays when the final lepton was an electron or a muon had been observed. When the lepton mass is different from zero, the relations (2.1) do not hold anymore, and experimental measurements of $B \rightarrow D^{(*)} \tau \nu$ decays are no more sensitive to one form factor only, even in the heavy quark limit. It has been recently observed that $B$ leptonic and semileptonic decays with a $\tau$ in the final state exhibit a tension of order $2 \sim 3 \sigma$ between experimental and theoretical results. Branching fraction measurements of $B^{-} \rightarrow \tau^{-} \bar{v}_{\tau}$ decay differ from the theoretical predictions based on the standard model fits, and the value of $\left|V_{u b}\right|$ represents the main source of theoretical uncertainty. BaBar collaboration has recently published results of their measurements of $B \rightarrow D^{(*)} \tau \nu$ branching fractions normalized to the corresponding $B \rightarrow D^{(*)} l \nu$ modes (with $l=e, \mu$ ) by using the full BaBar data sample [14]

$$
R(D)=\frac{\mathscr{B}(B \rightarrow D \tau v)}{\mathscr{B}(B \rightarrow D l v)}=0.440 \pm 0.072 \quad R\left(D^{\star}\right)=\frac{\mathscr{B}\left(B \rightarrow D^{*} \tau v\right)}{\mathscr{B}\left(B \rightarrow D^{*} l v\right)}=0.332 \pm 0.03
$$

where the statistical and systematic uncertainties have been combined in quadrature. The results (2.13) have been compared with the SM predictions, finding $R(D)_{\mathrm{S} M}=0.297 \pm 0.017$ and $R\left(D^{*}\right)_{\mathrm{S} M}=0.252 \pm 0.003$, averaged over electrons and muons. They respectively exceed the SM predictions by 2.0 and $2.7 \sigma$, corresponding to a combined discrepancy at the $3.4 \sigma$ level. A recent phenomenological approach questions the SM determination for $R(D)$ giving $R(D)=0.31 \pm 0.02$ [15] and a similar result, $R(D)=0.316 \pm 0.012 \pm 0.007$, is found in a recent full, $2+1$ flavor lattice QCD calculation [16]: both analysis reduce the significance of the discrepancy below $2 \sigma$. The values for $R(D)$ given by Belle [17] agree with those of BaBar, but have larger uncertainties. Current experimental measurements of $R(D)$ are statistics-limited, so the luminosities available at future flavor facilities should enable significant improvements. If the experimental results for $R\left(D^{(*)}\right)$ will be confirmed, they might point to new physics effects in semitauonic B decays. It has been excluded that the excess in Eqs. (2.13) over the SM can be explained within type II two Higgs doublet model [14] and in the context of the MSSM with MFV [18]. Instead, it is deemed possible in other SM extensions, e.g. in type III [19] and Aligned [20] two Higgs doublet models, by adopting effective Lagrangians [21, 22, 23], and so on.

\section{Inclusive $b \rightarrow u$ decays}

In inclusive $B \rightarrow X_{q} l v$ decays, we sum over all possible final states $X_{q}$, no matter if singleparticle or multi-particle states. Since inclusive decays do not depend on the details of final state, 
quark-hadron duality is generally assumed. In most of the phase space, long and short distance dynamics are factorized by means of the heavy quark expansion. However, the phase space region includes a region of singularity, also called endpoint or threshold region, plagued by the presence of large double (Sudakov-like) perturbative logarithms at all orders in the strong coupling ${ }^{1}$. For $b \rightarrow c$ semileptonic decays, the effect of the small region of singularity is not very important; in addition, corrections are not expected as singular as in the $b \rightarrow u$ case, being cut-off by the charm mass.

The analysis of inclusive charmless semileptonic decays is currently employed to determine the CKM parameter $\left|V_{u b}\right|$, which plays a crucial role in the study of the unitarity constraints and related questions. In principle, the method of extraction of $\left|V_{u b}\right|$ from inclusive $\bar{B} \rightarrow X_{u} l \bar{v}_{l}$ decays follows in the footsteps of the $\left|V_{c b}\right|$ determination from $\bar{B} \rightarrow X_{c} l \bar{v}_{l}$, that employs the OPE expansion in the systematic framework provided by the heavy quark effective theory. However, in $\bar{B} \rightarrow X_{u} l \bar{v}_{l}$ decays, the copious background from the $\bar{B} \rightarrow X_{c} l \bar{v}_{l}$ process, which has a rate about 50 times higher, stands in the way, and the experimental sensitivity is higher in restricted regions of phase space, where such background is highly suppressed by kinematics. They include the threshold region, where the previous approch fails, and new theoretical issues need to be addressed. A lot of effort has been devoted to enlarge the experimental range, so to reduce on the whole the weight of the endpoint region. Latest results by Belle [30] access about the $90 \%$ of the $\bar{B} \rightarrow X_{u} l \bar{v}_{l}$ phase space, claiming an overall uncertainty of $7 \%$ on $\left|V_{u b}\right|$. A similar portion of the phase space is covered also by the more recent BaBar analysis [31]. On the theoretical side several approaches have been devised to analyze data in the threshold region, with differences in treatment of perturbative corrections and the parameterization of nonperturbative effects.

Experimental results are provided by CLEO, BaBar and Belle; the B factories have provided the largest and more recent amount of data. The average values for $\left|V_{u b}\right|$ can be extracted from the partial branching fractions, adopting a specific theoretical framework and taking into account correlations among the various measurements and theoretical uncertainties. The averaging procedure has been developed by HFAG [3], that has also checked the consistency among inputs of the different theoretical frameworks. In Table 1 we list the HFAG averages, that are determined by comparing the measured partial branching fractions in selected regions of phase-space to theoretical calculations of the corresponding rates. We have listed all the QCD theoretical calculations that take into account the whole set of experimental results, or most of it, starting from 2002 CLEO data [32]. Very roughly speaking, the BLNP approach by Bosch, Lange, Neubert, and Paz [33] and the GGOU one by Gambino, Giordano, Ossola and Uraltsev [34] differ in their treatment of Sudakov double logarithms resumming and subleading shape function modeling. The DGE, the dressed gluon exponentiation, by Andersen and Gardi $[35,36]$ and the ADFR approach, by Aglietti, Di Lodovico, Ferrara, and Ricciardi, [37, 38, 39] start analyzing the singularities of the perturbative expansion in moment space; in the ADFR approach, an effective running coupling is introduced to help estimating nonperturbative corrections. The results listed in Table 1 are consistent within the errors, but the theoretical uncertainty among determinations can reach 10\%. Other solutions have been proposed in [40, 41, 42].

Notwithstanding all the experimental and theoretical efforts, the values of $\left|V_{u b}\right|$ extracted from

\footnotetext{
${ }^{1}$ for theoretical aspects of threshold resummation in $B$ decays see e.g. [24, 25, 26, 27, 28, 29].
} 


\begin{tabular}{lc}
\hline \hline Theory & $\left|V_{u b}\right| \times 10^{3}$ \\
\hline \hline BLNP & $4.40 \pm 0.15_{-0.21}^{+0.19}$ \\
\hline DGE & $4.45 \pm 0.15_{-0.16}^{+0.15}$ \\
\hline ADFR & $4.03 \pm 0.13_{-0.12}^{+0.18}$ \\
\hline GGOU & $4.39 \pm 0.15_{-0.20}^{+0.12}$ \\
\hline \hline
\end{tabular}

Table 1: Comparison of inclusive determinations of $\left|V_{u b}\right|[3]$.

inclusive decays maintain about two $\sigma$ above the values given by exclusive determinations. The most recent BaBar estimate from exclusive decays yields $\left|V_{u b}\right|=(3.25 \pm 0.31) \times 10^{-3}$ [43] and it is determined from the simultaneous fit to the experimental data and the lattice theoretical predictions. It is compatible with the Belle result [44] of $\left|V_{u b}\right|=(3.43 \pm 0.33) \times 10^{-3}$. Also indirect fits prefer a lower value of $\left|V_{u b}\right|$, such as $\left|V_{u b}\right|=(3.62 \pm 0.14) \times 10^{-3}$ by the UTfit Collaboration [45].

\section{References}

[1] G. Ricciardi, Brief review on semileptonic B decays, Mod. Phys. Lett. A 27 (2012) 1230037 [arXiv:1209.1407 [hep-ph]].

[2] G. Ricciardi, Semileptonic B and D decays-latest developments in proceedings of the 10th International Conference on Flavor Physics and CP Violation, Hefei, China, May 21-25, 2012 arXiv:1209.5650 [hep-ph].

[3] Y. Amhis et al. [Heavy Flavor Averaging Group Collaboration], Averages of b-hadron, c-hadron, and tau-lepton properties as of early 2012, arXiv:1207.1158 [hep-ex].

[4] C. Bernard, C. E. De Tar, M. Di Pierro, A. X. El-Khadra, R. T. Evans, E. D. Freeland, E. Gamiz and S. Gottlieb et al., The $\bar{B} \rightarrow D^{*} \ell \bar{v}$ form factor at zero recoil from three-flavor lattice QCD: A Model independent determination of $\left|V_{c b}\right|$, Phys. Rev. D 79 (2009) 014506 [arXiv:0808.2519 [hep-lat]].

[5] J. A. Bailey et al. [Fermilab Lattice and MILC Collaboration], $B \rightarrow D^{*} l v$ at zero recoil: an update, POS (LATTICE2010) 311. [arXiv:1011.2166 [hep-lat]].

[6] P. Gambino, T. Mannel and N. Uraltsev, $B \rightarrow D^{*}$ at zero recoil revisited, Phys. Rev. D 81 (2010) 113002 [arXiv:1004.2859 [hep-ph]].

[7] P. Gambino, T. Mannel and N. Uraltsev, $B \rightarrow D^{*}$ Zero-Recoil Formfactor and the Heavy Quark Expansion in QCD: A Systematic Study, JHEP 1210 (2012) 169 [arXiv:1206.2296 [hep-ph]].

[8] M. Okamoto, C. Aubin, C. Bernard, C. E. DeTar, M. Di Pierro, A. X. El-Khadra, S. Gottlieb and E. B. Gregory et al., Semileptonic $D \rightarrow \pi / K$ and $B \rightarrow \pi / D$ decays in $2+1$ flavor lattice $Q C D$, Nucl. Phys. Proc. Suppl. 140 (2005) 461 [hep-lat/0409116].

[9] G. M. de Divitiis, E. Molinaro, R. Petronzio and N. Tantalo, Quenched lattice calculation of the $B \rightarrow$ Dlv decay rate, Phys. Lett. B 655 (2007) 45 [arXiv:0707.0582 [hep-lat]].

[10] G. M. de Divitiis, R. Petronzio and N. Tantalo, Quenched lattice calculation of semileptonic heavy-light meson form factors, JHEP 0710 (2007) 062 [arXiv:0707.0587 [hep-lat]].

[11] B. Aubert et al. [BABAR Collaboration], Measurement of $\left|V_{c b}\right|$ and the Form-Factor Slope in $\bar{B} \rightarrow$ Dlv Decays in Events Tagged by a Fully Reconstructed B Meson, Phys. Rev. Lett. 104, (2010) 011802 [arXiv:0904.4063 [hep-ex]]. 
[12] N. Uraltsev, A 'BPS' expansion for B and D mesons, Phys. Lett. B 585 (2004) 253 [hep-ph/0312001].

[13] J. Beringer et al. [Particle Data Group Collaboration], Review of Particle Physics (RPP), Phys. Rev. D 86, (2012) 010001.

[14] J. P. Lees et al. [BaBar Collaboration], Evidence for an excess of $\bar{B} \rightarrow D^{(*)} \tau^{-} \bar{v}_{\tau}$ decays, Phys. Rev. Lett. 109, 101802 (2012) [arXiv:1205.5442 [hep-ex]].

[15] D. Becirevic, N. Kosnik and A. Tayduganov, $\bar{B} \rightarrow D \tau \bar{v}_{\tau} v s . \bar{B} \rightarrow D \mu \bar{v}_{\mu}$, Phys. Lett. B 716 (2012) 208 [arXiv:1206.4977 [hep-ph]].

[16] J. A. Bailey, A. Bazavov, C. Bernard, C. M. Bouchard, C. DeTar, D. Du, A. X. El-Khadra and J. Foley et al., Refining new-physics searches in $B->D \tau v$ decay with lattice $Q C D$, Phys. Rev. Lett. 109 (2012) 071802 [arXiv:1206.4992 [hep-ph]].

[17] I. Adachi et al. [Belle Collaboration], Measurement of $B \rightarrow D^{(*)} \tau v$ using full reconstruction tags, arXiv:0910.4301 [hep-ex].

[18] W. Altmannshofer, M. Carena, N. Shah and F. Yu, Indirect Probes of the MSSM after the Higgs Discovery, arXiv:1211.1976 [hep-ph].

[19] A. Crivellin, C. Greub and A. Kokulu, Explaining $B \rightarrow D \tau \nu, B \rightarrow D^{*} \tau \nu$ and $B \rightarrow \tau \nu$ in a $2 H D M$ of type III, Phys. Rev. D 86 (2012) 054014 [arXiv:1206.2634 [hep-ph]].

[20] A. Celis, M. Jung, X. -Q. Li and A. Pich, Sensitivity to charged scalars in $B \rightarrow D^{(*)} \tau v_{\tau}$ and $B \rightarrow \tau v_{\tau}$ decays, JHEP 1301 (2013) 054 [arXiv:1210.8443 [hep-ph]].

[21] S. Fajfer, J. F. Kamenik and I. Nisandzic, On the $B \rightarrow D^{*} \tau \bar{v}_{\tau}$ Sensitivity to New Physics, Phys. Rev. D 85 (2012) 094025 [arXiv:1203.2654 [hep-ph]].

[22] S. Fajfer, J. F. Kamenik, I. Nisandzic and J. Zupan, Implications of Lepton Flavor Universality Violations in B Decays, Phys. Rev. Lett. 109 (2012) 161801 [arXiv:1206.1872 [hep-ph]].

[23] A. Datta, M. Duraisamy and D. Ghosh, Diagnosing New Physics in $b \rightarrow c \tau v_{\tau}$ decays in the light of the recent BaBar result, Phys. Rev. D 86 (2012) 034027 [arXiv:1206.3760 [hep-ph]].

[24] U. Aglietti and G. Ricciardi, Approximate NNLO threshold resummation in heavy flavor decays, Phys. Rev. D 66 (2002) 074003 [hep-ph/0204125].

[25] U. Aglietti, G. Ricciardi and G. Ferrera, Threshold resummed spectra in $B \rightarrow X_{u} l v$ decays in $N L O(I)$, Phys. Rev. D 74 (2006) 034004 [hep-ph/0507285].

[26] U. Aglietti, G. Ricciardi and G. Ferrera, Threshold resummed spectra in $B \rightarrow X_{u} l v$ decays in NLO (II), Phys. Rev. D 74 (2006) 034005 [hep-ph/0509095].

[27] U. Aglietti, G. Ricciardi and G. Ferrera, Threshold resummed spectra in $B \rightarrow X_{u} l v$ decays in NLO (III), Phys. Rev. D 74 (2006) 034006 [hep-ph/0509271].

[28] U. Aglietti, L. Di Giustino, G. Ferrera, A. Renzaglia, G. Ricciardi and L. Trentadue, Threshold Resummation in $B \rightarrow X_{c} l v_{l}$ Decays, Phys. Lett. B 653 (2007) 38 [arXiv:0707.2010 [hep-ph]].

[29] L. Di Giustino, G. Ricciardi and L. Trentadue, Minimal prescription corrected spectra in heavy quark decays, Phys. Rev. D 84 (2011) 034017 [arXiv:1102.0331 [hep-ph]].

[30] P. Urquijo et al. [Belle Collaboration], Measurement Of $\left|V_{u b}\right|$ From Inclusive Charmless Semileptonic B Decays, Phys. Rev. Lett. 104 (2010) 021801 [arXiv:0907.0379 [hep-ex]].

[31] J. P. Lees et al. [BABAR Collaboration], Study of $\bar{B} \rightarrow X_{u} \ell \bar{v}$ decays in $B \bar{B}$ events tagged by a fully reconstructed B-meson decay and determination of $\left|V_{u b}\right|$, Phys. Rev. D 86 (2012) 032004 [arXiv:1112.0702 [hep-ex]]. 
[32] A. Bornheim et al. [CLEO Collaboration], Improved measurement of $|V(u b)|$ with inclusive semileptonic B decays, Phys. Rev. Lett. 88 (2002) 231803 [hep-ex/0202019].

[33] B. O. Lange, M. Neubert and G. Paz, Theory of charmless inclusive B decays and the extraction of $V(u b)$, Phys. Rev. D 72 (2005) 073006 [hep-ph/0504071].

[34] P. Gambino, P. Giordano, G. Ossola and N. Uraltsev, Inclusive semileptonic B decays and the determination of $|V(u b)|$, JHEP 0710 (2007) 058 [arXiv:0707.2493 [hep-ph]].

[35] J. R. Andersen and E. Gardi, Inclusive spectra in charmless semileptonic B decays by dressed gluon exponentiation, JHEP 0601 (2006) 097 [hep-ph/0509360].

[36] E. Gardi, On the determination of $|V(u b)|$ from inclusive semileptonic B decays, arXiv:0806.4524 [hep-ph].

[37] U. Aglietti and G. Ricciardi, A Model for next-to-leading order threshold resummed form-factors, Phys. Rev. D 70 (2004) 114008 [hep-ph/0407225].

[38] U. Aglietti, G. Ferrera and G. Ricciardi, Semi-Inclusive B Decays and a Model for Soft-Gluon Effects, Nucl. Phys. B 768 (2007) 85 [hep-ph/0608047].

[39] U. Aglietti, F. Di Lodovico, G. Ferrera and G. Ricciardi, Inclusive measure of $\left|V_{u b}\right|$ with the analytic coupling model, Eur. Phys. J. C 59 (2009) 831 [arXiv:0711.0860 [hep-ph]].

[40] C. W. Bauer, Z. Ligeti and M. E. Luke, Precision determination of $|V(u b)|$ from inclusive decays, Phys. Rev. D 64 (2001) 113004 [hep-ph/0107074].

[41] A. K. Leibovich, I. Low and I. Z. Rothstein, Extracting V(ub) without recourse to structure functions, Phys. Rev. D 61 (2000) 053006 [hep-ph/9909404].

[42] Z. Ligeti, I. W. Stewart and F. J. Tackmann, Treating the b quark distribution function with reliable uncertainties, Phys. Rev. D 78 (2008) 114014 [arXiv:0807.1926 [hep-ph]].

[43] J. P. Lees et al. [BABAR Collaboration], Branching fraction and form-factor shape measurements of exclusive charmless semileptonic $B$ decays, and determination of $\left|V_{u b}\right|$, Phys. Rev. D 86 (2012) 092004 [arXiv:1208.1253 [hep-ex]].

[44] H. Ha et al. [BELLE Collaboration], Measurement of the decay $B^{0} \rightarrow \pi^{-} \ell^{+} v$ and determination of $\left|V_{u b}\right|$, Phys. Rev. D 83 (2011) 071101 [arXiv:1012.0090 [hep-ex]].

[45] C. Tarantino, Flavor Lattice QCD in the Precision Era, arXiv:1210.0474 [hep-ph]. 